\title{
Prototyping a Dual-Channel Receiver for use in a Staring Cooperative Radar Network for the Detection of Drones
}

\author{
Benjamin Griffin*, Alessio Balleri*, Chris Baker**, Mohammed Jahangir** \\ ${ }^{*}$ Centre for Electronic Warfare, Information and Cyber, Cranfield University \\ Defence Academy of the UK, Shrivenham, SN6 8LA \\ email: b.griffin@cranfield.ac.uk, a.balleri@cranfield.ac.uk \\ ${ }^{* *}$ School of Engineering, University of Birmingham \\ Birmingham, B15 2TT, United Kingdom \\ email: c.j.Baker.1@bham.ac.uk,m.jahangir@bham.ac.uk
}

\begin{abstract}
Staring radars use a transmitting static wide-beam antenna and a directive digital array to form multiple simultaneous beams on receive. Because beams are fixed, the radar can employ long integration times to detect slow low-RCS targets, such as drones, which present a challenge to traditional air surveillance radar. The use of multiple spatially separated receivers cooperating with the staring transmitters in a multistatic network allows multi-perspective target acquisitions that can help mitigate interference, such as signal multipath, and ultimately enhance the detection of drones and reduce target parameter estimation errors. Here, the design of a dual-channel receiver prototype for use in a multistatic cooperative network is presented. Several measurements have been taken using the prototype receiver in a bistatic configuration to test and assess its performance.
\end{abstract}

\section{Introduction}

The robust detection of drones is an active area of research in the radar domain. Due to their small size, low flight profile and slow speed, drones present a unique challenge to traditional air surveillance radars. Studies into the use of radar networks are receiving increased interest as they have the potential to improve the detection of drones and drone-like targets [1]. A proposed list of capabilities for a radar system to be able to detect and track drones has been presented by Poitevin et al [2] and include:

- Sensitivity for very small detectable signature

- Fast refresh rate

- Height measurement capability
- Large volumetric coverage requirements

- Nuisance elimination

- System cost

Staring array radar have been built that can detect small drones out to a range of $5 \mathrm{~km}$ and satisfy the sensitivity, fast refresh rate and height measurement capability [3]. The combination of the staring array radar and a multistatic radar network has the potential to meet the requirements for a drone detection and tracking system. 
Small scale multistatic radar networks have successfully demonstrated an ability to detect and track drones; in particular, the NetRad and NeXtRAD radar [4] [5]. These are active multistatic radar networks operating in the $\mathrm{L}$ and X-band. Both the NetRad and NeXtRAD radar use identical nodes, whereas the network proposed here consists of a central staring radar and multiple multistatic receive-only nodes cooperating with the central radar. These networks have also shown the capability to detect the micro-Doppler signature of a drones rotors, which is a crucial part of improving the classification performance of a radar system [6] [7]. Distributed radar networks consisting of multiple homogeneous radars can be built using low-cost commercial off the shelf (COTS) hardware [8]. Guo et al presented a distributed radar network in which a K-band Continuous-Wave $(\mathrm{CW})$ transceiver was developed to use a targets micro-Doppler signatures for detection and classification of targets [9].

This paper presents the design and results of experimental trials with a prototype dual-channel receiver. The receiver is designed to cooperate with an Aveillant radar as part of a multistatic radar network. The Aveillant Gamekeeper radar is a staring array radar designed to detect drones and transmits a pulsed waveform in the L-band [10]. The goal of the network is to improve the detection of drones, reduce estimation/localisation errors, and mitigate the effects of signal multipath. An initial study into the optimal geometry cooperative staring radar network has been previously explored as part of this project [11].

\section{Receiver Design}

The network's receivers are designed using passive radar principles with each receiver utilising two channels. The use of two channels allows the receivers to record the transmitted signal, the reference channel, from the Aveillant radar and the reflections from the target simultaneously, the surveillance channel. The Global Positioning System (GPS) is used to help synchronise data across the network. Each receiver in the network is connected to a GPS antenna, which the computer can use to record the receivers position and the current UTC time. Most modern drones also contain a GPS unit used to record aspects of the drone's flight such as its position, speed and heading. After a cooperative trial is complete, the drone's position can be more accurately correlated with the data recorded at each receiver.

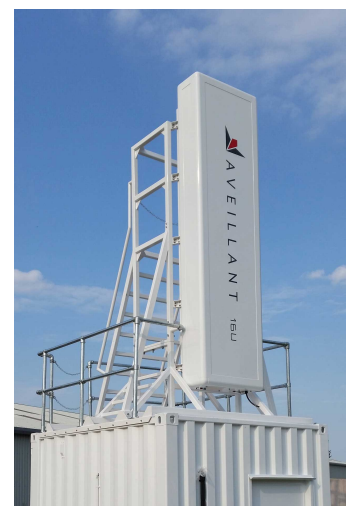

(a)

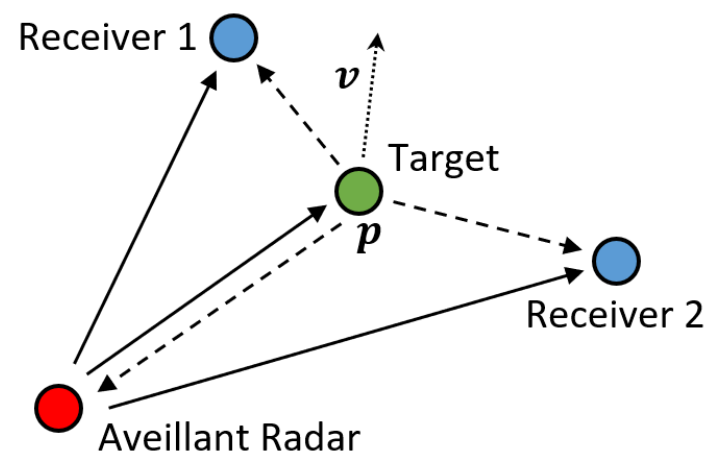

(b)

Figure 1: Aveillant Gamekeeper Radar (a), Proposed Multistatic Radar Network (b) 


\section{Receiver Implementation}

The dual-channel receiver prototype consists of two receiver blocks, a VCO block, digitizer, GPS antenna and computer, as shown in Fig. 2. The VCO is an Aaren Technology Electronics RFF25611 generates the LO signal at L-band, necessary to match the carrier frequency used by the Aveillant radar. The LO signal is amplified (mini-circuit ZX60-P105LN+) and attenuated to $10 \mathrm{~dB}$. An RF splitter (mini-circuit ZX10-2-20-S+) splits the VCO signal into two signals at $7 \mathrm{~dB}$, necessary to drive the mixers on the receiving channels.

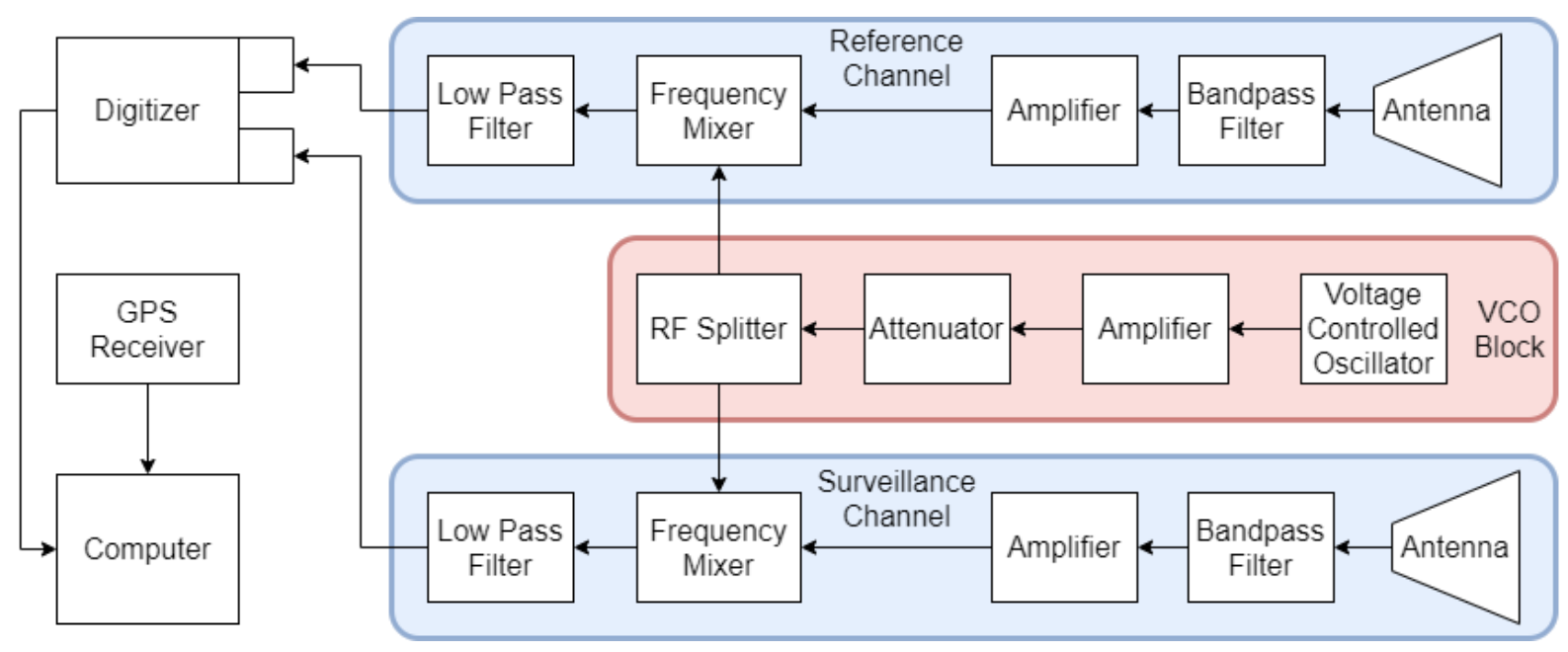

Figure 2: Dual Channel Receiver Block Diagram

The received signal from each antenna is filtered by a bandpass filter and then is amplified by a low noise amplifier (mini-circuit ZX60-P162LN+) to increase the signal power by $22 \mathrm{~dB}$. The amplified signal is mixed down (mini-circuit ZX10-2-20-S+) using the LO signal from the VCO block. The received signals, now at baseband, is filtered by a $45 \mathrm{MHz}$ low pass filter (mini-circuit VLF-45+) and digitized using a TiePie Handyscope HS6D digital oscilloscope. The data from the digitizer is recorded by the computer along with the current UTC time as well as the receiver's latitude and longitude. The timing and positioning data is obtained from the attached GPS, a GlobalSat BU-353-S4.

\section{Signal Processing}

The radar operates in a block data collection cycle. The computer reads the receivers Latitude, Longitude and current time (UTC) from the attached GPS receiver and triggers the digitizer. The digitizer is preconfigured to record a continuous block of data from both channels when triggered. Once the digitizer has finished recording, the computer saves the data to disk along with the information from the GPS and the digitizer's settings. Once all the data is saved, the computer triggers the next measurement.

Each block of data is loaded and processed independently. The data from both channels are loaded as two vectors, and the Hilbert transform used to recover the IQ components of the 
signal. The reference channel's peak in the frequency domain is selected and used to place a bandpass filter over the main lobe of the signal. The filtered signals are transformed back to the time domain and, each channel is divided into pulses to form two Fast-Time Slow-Time (FTST) matrices. The surveillance FTST data is matched filtered with the FTST data from the reference channel. The short-time Fourier transform and the Range-Doppler map can then be generated. Moving Target Indication (MTI) is performed on the Range-Doppler map to remove the direct transmitter to receiver signal and stationary clutter.

\section{Initial Measurements}

Initial measurements of a rotating fan and the rotors of a remotely controlled helicopter were carried out to evaluate the performance of the dual-channel receiver prototype, at the Radar Laboratory of Cranfield University, at the Defence Academy of the UK in Shrivenham. During the measurements only one bandpass filter was available, installed on the reference channel. In both cases the range-Doppler response of the target, after zero Doppler removal, and the Short-Time Fourier Transforms (STFT) are generated.

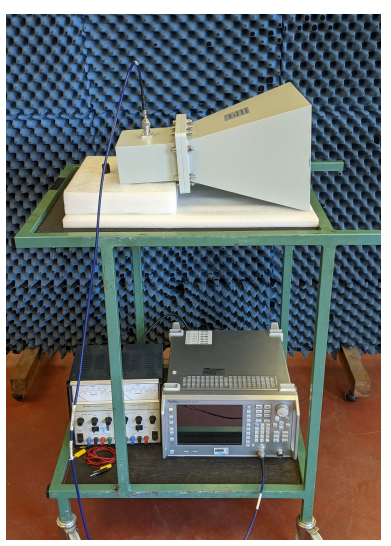

(a)

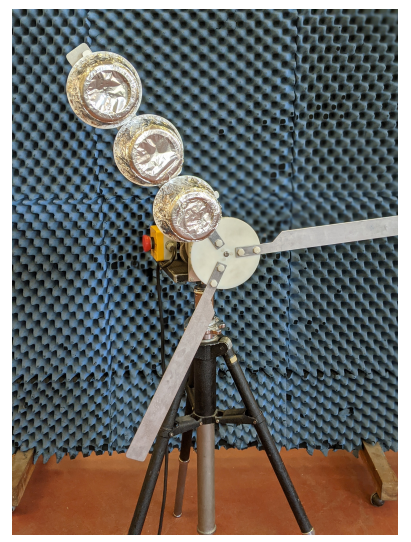

(b)

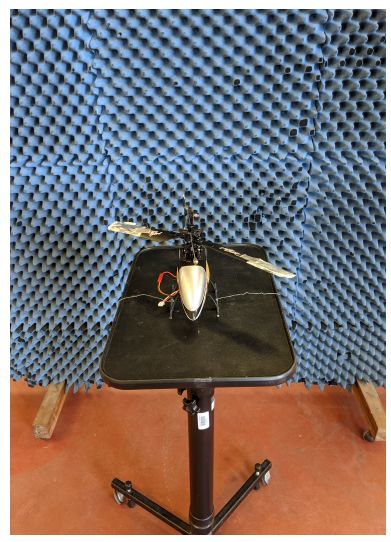

(c)

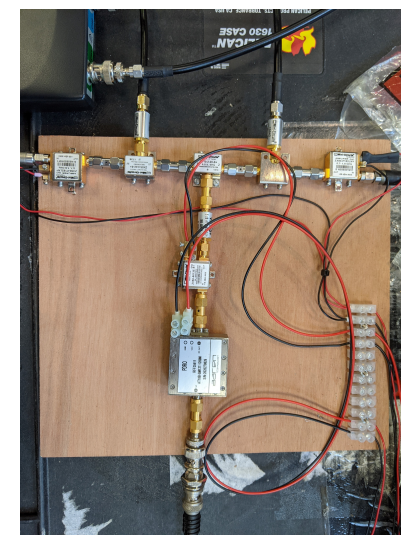

(d)

Figure 3: Transmitter (a), Rotating Fan (b), Helicopter (c), Receiver Hardware (d)

A separate transmitter consisting of a signal generator, an Anritsu MS2691A, and antenna, an A-Info LB-OH-650-10, was placed off to the side oriented towards the target Fig. 3 (a). Two broadband horn antennas (A-Info LB-7810) were connected to both the reference and surveillance channel. The prototype receiver was placed such that the reference antenna could record the transmitter's signal, and the surveillance antenna could observe the target without picking up the direct signal from the transmitter as shown in Fig. 3 (d). Experimental results were obtained using an integration period of $250 \mathrm{~ms}$ sampled at $20 \mathrm{MHz}$ ( $5 \mathrm{M}$ total samples). The frequency of the VCO was tuned to $5 \mathrm{MHz}$ below the centre frequency of the transmitted signal. 


\subsection{Measurements of Rotating Fan}

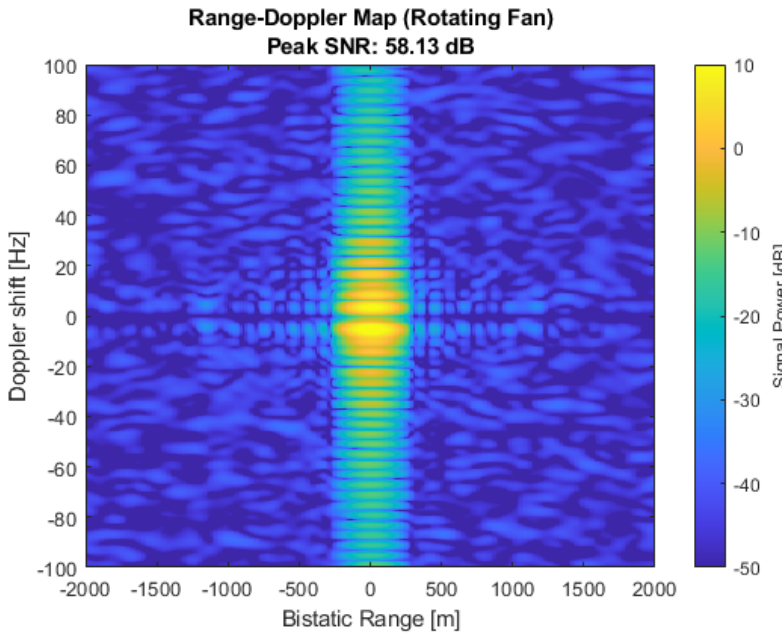

(a)

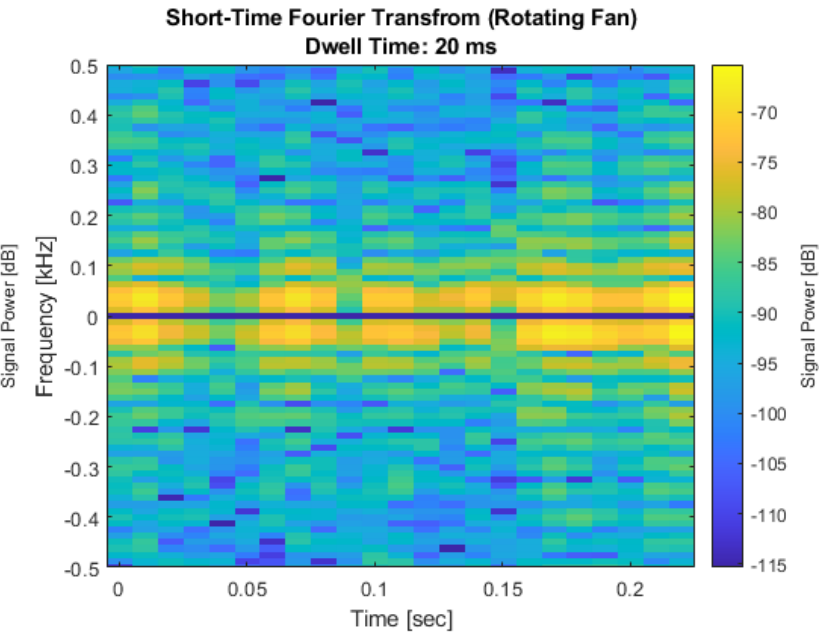

(b)

Figure 4: Range-Doppler Map (a) and Short-Time Fourier Transform (b) of the Rotating Fan

Fig. 4 shows the measurements of a rotating fan taken by the dual-channel receiver. The fan consists of three $46 \mathrm{~cm}$ long blades rotating at approximately 120 rotations per minute (rpm) Fig. 3 (b). Fig. 4 (a) shows the range-Doppler response of the rotating fan. The fan is detected with a peak SNR of $54 \mathrm{~dB}$ at a range of $7.5 \mathrm{~m}$. Two peaks are visible at $\pm 5 \mathrm{~Hz}$ along with multiple range and Doppler side lobes. Fig. 4 (b) shows the STFT of the target using a dwell time of $20 \mathrm{~ms}$, which is starting to show the periodic motion of the fan, however further work is required to improve this image.

\subsection{Measurements of a Remote Control Helicopter's Rotors}

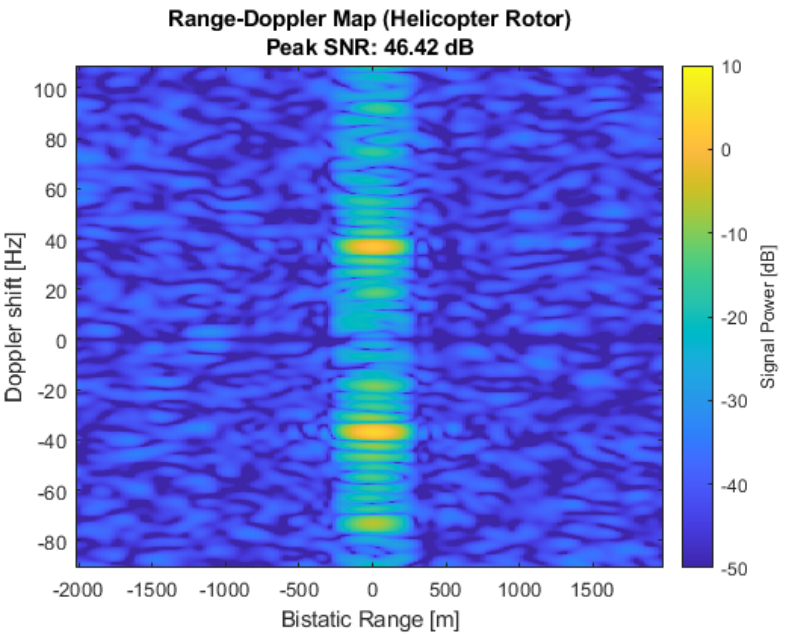

(a)

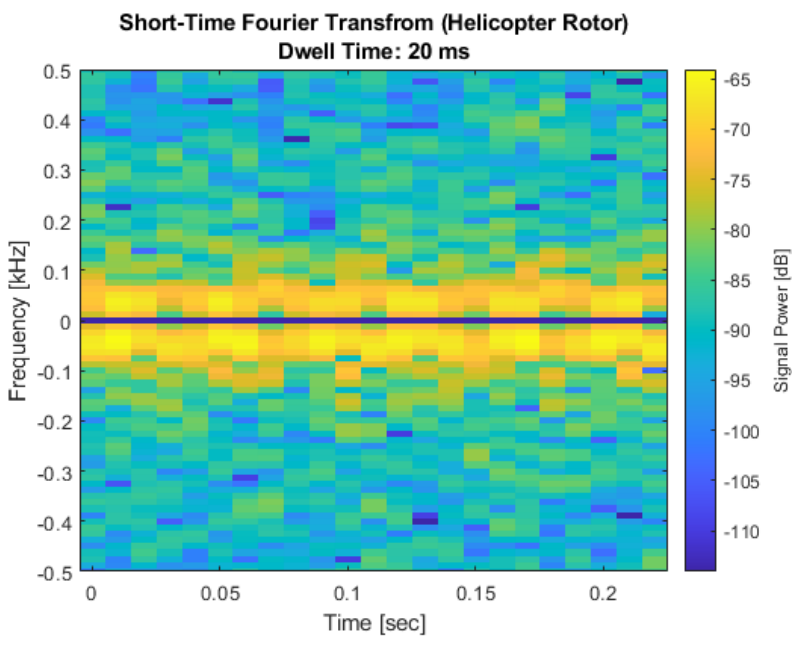

(b)

Figure 5: Range-Doppler Map (a) and Short-Time Fourier Transform (b) of the Helicopters Rotors 
Fig. 5 shows the measurements of a remote control helicopters rotors taken by the dual-channel receiver. The helicopter with $18.5 \mathrm{~cm}$ long rotors was placed on a stand with the rotors spinning in a stationary position Fig. 3 (c). Results in Fig. 5 (a) shows the range-Doppler response of the Helicopters rotors. Two peaks are visible with a Doppler shift of $\pm 40 \mathrm{~Hz}$, the rotors are detected with a peak SNR of $46 \mathrm{~dB}$ at a range of $7.5 \mathrm{~m}$. Fig. 4 (b) shows the STFT of the target using a dwell time of $20 \mathrm{~ms}$, the periodic signature of the rotors is visible showing multiple rotor rotations, indicating a maximum Doppler shift of $625 \mathrm{~Hz}$.

\section{Conclusion}

The purpose of this project is to develop and prototype an L-band multistatic receiver to support further measurements as part of a small scale multistatic radar network centred on a staring radar. The design and initial testing of the receiver have been presented as well as initial measurements of a rotating fan and the rotors of a remotely controlled helicopter. Both targets have been shown to be detectable in the range-Doppler domain. The STFT can partially extract the periodic signatures of both targets, with the helicopter's rotors' signature being the clearest. The installation of the second bandpass filter on the surveillance channel should improve these results. Further measurements of drones in flight and at range are planned involving an Aveillant radar and multiple dual-channel receivers.

\section{Acknowledgements}

The authors thank Aveillant Ltd, a Thales company and Cranfield Defence and Security for funding this work under the Cranfield University 50:50 Industrial PhD scheme. The authors would also like to thank Janko Scepanovic for his initial work on the design.

\section{References}

[1] H. Griffiths. Developments in bistatic and networked radar. Proceedings of 2011 IEEE CIE International Conference on Radar, RADAR 2011, 1:10-13, 2011.

[2] P. Poitevin, M. Pelletier, and P. Lamontagne. Challenges in detecting UAS with radar. In Proceedings - International Carnahan Conference on Security Technology, volume 2017-Octob, pages 1-6. IEEE, oct 2017.

[3] M. Jahangir and C.J. Baker. Persistence surveillance of difficult to detect micro-drones with Lband 3-D holographic radar ${ }^{\mathrm{TM}}$. In 2016 CIE International Conference on Radar, RADAR 2016, pages 1-5. IEEE, oct 2017.

[4] F. Hoffmann, M. Ritchie, F. Fioranelli, A. Charlish, and H. Griffiths. Micro-Doppler based detection and tracking of UAVs with multistatic radar. 2016 IEEE Radar Conference, RadarConf 2016, pages 1-6, 2016.

[5] R. Palama, F. Fioranelli, M. Ritchie, M.R. Inggs, S. Lewis, and H. Griffiths. Measurements of Multistatic XL Band Radar Signatures of UAVS. In 2019 International Radar Conference, RADAR 2019, pages 1-5. IEEE, sep 2019.

[6] F. Fioranelli, M. Ritchie, H. Griffiths, and H. Borrion. Classification of loaded/unloaded microdrones using multistatic radar. Electronics Letters, 51(22):1813-1815, 2015. 
[7] M. Ritchie, F. Fioranelli, H. Griffiths, and B. Torvik. Monostatic and bistatic radar measurements of birds and micro-drone. In 2016 IEEE Radar Conference, RadarConf 2016, pages 1-5. IEEE, may 2016.

[8] X. Guo, C. Ng, E. de Jong, and A. Smits. Concept of Distributed Radar System for mini-UAV Detection in Dense Urban Environment. In 2019 International Radar Conference (RADAR), pages 1-4. IEEE, sep 2020.

[9] X. Guo, C. Ng, E. de Jong, and A. Smits. Micro-doppler based mini-UAV detection with lowcost distributed radar in dense urban environment. In EuRAD 2019 - 2019 16th European Radar Conference, pages 189-192, 2019.

[10] M. Jahangir and Chris.J. Baker. Extended dwell Doppler characteristics of birds and micro-UAS at l-band. In Proceedings International Radar Symposium, pages 1-10. IEEE, jun 2017.

[11] B. Griffin, A. Balleri, C.J. Baker, and M. Jahangir. Optimal receiver placement in staring cooperative radar networks for detection of drones. In IEEE National Radar Conference - Proceedings, volume 2020-Septe, pages 1-6. IEEE, sep 2020. 